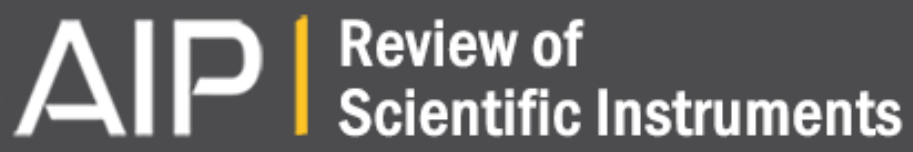

\section{High pressure hydrogen loading cell for photoconductivity measurements down to the milliKelvin regime}

\author{
A. F. Th. Hoekstra, T. F. Rosenbaum, and A. S. Roy
}

Citation: Review of Scientific Instruments 73, 119 (2002); doi: 10.1063/1.1425775

View online: http://dx.doi.org/10.1063/1.1425775

View Table of Contents: http://scitation.aip.org/content/aip/journal/rsi/73/1?ver=pdfcov

Published by the AIP Publishing

\section{Articles you may be interested in}

Development of loading system for liquid hydrogen into diamond-anvil cells under low temperature

Rev. Sci. Instrum. 81, 033901 (2010); 10.1063/1.3321480

Miniature diamond anvil cell for broad range of high pressure measurements

Rev. Sci. Instrum. 80, 043906 (2009); 10.1063/1.3122051

Highly efficient gaseous sample loading technique for diamond anvil cells

Rev. Sci. Instrum. 75, 5149 (2004); 10.1063/1.1813111

A combined high-pressure reaction cell-ultrahigh vacuum chamber with sample transfer system

Rev. Sci. Instrum. 69, 2757 (1998); 10.1063/1.1149011

He activated loading device for low temperature uniaxial and anvil cell pressure experiments

Rev. Sci. Instrum. 68, 3120 (1997); 10.1063/1.1148254

\section{AlP hameded theses}

Journal of Applied Physics is pleased to announce André Anders as its new Editor-in-Chief 


\title{
High pressure hydrogen loading cell for photoconductivity measurements down to the milliKelvin regime
}

\author{
A. F. Th. Hoekstra \\ The James Franck Institute, The University of Chicago, Chicago, Illinois 60637 and Condensed Matter \\ Physics, Faculty of Exact Sciences, Vrije Universiteit, Amsterdam, The Netherlands \\ T. F. Rosenbaum ${ }^{\text {a) }}$ and A. S. Roy \\ The James Franck Institute and Department of Physics, The University of Chicago, Chicago, Illinois 60637
}

(Received 23 July 2001; accepted for publication 25 September 2001)

\begin{abstract}
A gas loading cell has been developed to load rare earth thin film samples with hydrogen at pressures up to 200 bars at room temperature. A miniature valve closes the gas inlet, after which the cell is suspended from the cold tail of a ${ }^{3} \mathrm{He}$ flow cryostat into the bore of a $16 \mathrm{~T}$ superconducting magnet. An ultraviolet stroboscope outside the cryostat illuminates the sample by way of an optical fiber to a window in the cell. Electrical feedthroughs permit photoconductivity and magnetotransport measurements over three decades in temperature. Extension to other materials, different gas atmospheres, and helium dilution refrigerator temperatures is straightforward.

(C) 2002 American Institute of Physics. [DOI: 10.1063/1.1425775]
\end{abstract}

\section{INTRODUCTION}

The fundamental properties of a wide variety of materials can be probed and even altered under optical illumination. In addition to standard light scattering techniques for characterization, optical exposure can create charge carriers via excitations over an insulating gap or induce structural changes via subtle rearrangements of chemical bonds. These physical and electronic changes can fine tune a material's technological features, or with the added flexibility to control atmosphere, temperature, and applied field, may permit high resolution studies of phase transformations.

We report here the design of an optical cell with electrical feedthroughs that can accommodate high gas pressures and can be cooled to milliKelvin temperatures in a high magnetic field. Our motivation was the investigation of the metal-insulator transition in rare earth hydride films. From their discovery, the remarkable reversible optical and electronic properties of these materials at room temperature upon hydrogen loading ${ }^{1}$ have been assumed to reflect a continuous metal-insulator transition at the absolute zero of temperature. We tested ${ }^{2}$ this assumption for the first time in the case of a $500 \mathrm{~nm}$ thick polycrystalline yttrium hydride $\mathrm{YH}_{x}$ film covered by $5 \mathrm{~nm}$ of $\mathrm{Pd}$, the prototype of the so-called switchable mirrors. These experiments required a hydrogen atmosphere to open the insulating gap, ultraviolet illumination at temperatures below $T=10 \mathrm{~K}$ to create persistent photocarriers, and magnetotransport measurements in the $T=0$ limit to reveal the quantum critical behavior.

The high-pressure hydrogen loading cell for photoconductivity measurements was mounted in a ${ }^{3} \mathrm{He}$ flow cryostat equipped with a $16 \mathrm{~T}$ superconducting magnet (Oxford Instruments Heliox system, $100 \mu \mathrm{W}$ cooling power at a base temperature $\sim 300 \mathrm{mK}$ ). It would be possible as well to sus-

${ }^{\text {a)} E l e c t r o n i c ~ m a i l: ~ t-r o s e n b a u m @ u c h i c a g o . e d u ~}$ pend the cell from the mixing chamber of a helium dilution refrigerator into the bore of a superconducting magnet (minimum $50 \mathrm{~mm}$ bore) without modification. The following requirements were taken into account in the design of the cell. (i) At room temperature (RT) the cell withstands internal gas pressure of at least 200 bars (the limit of commercially available gas containers, which provide the pressurized hydrogen without the need of a compressor). It is leak tight down to the lowest testable level both under vacuum conditions and for pressures up to 200 bars, and it remains so during temperature sweeps between RT and base temperature (in the course of which the internal pressure is lower than at RT). (ii) The cell materials are nonmagnetic, nonsuperconducting, have a small specific heat, and are not embrittled by hydrogen absorption. (iii) Sufficient thermal conduction from the ${ }^{3} \mathrm{He}$ pot to the sample is provided even at the lowest $T$ and the highest magnetic field $H$ via a cold tail and the cell, which is suspended in the inner vacuum chamber (IVC) of the ${ }^{3} \mathrm{He}$ flow cryostat and inside the bore of the magnet. Eddy currents should be reduced to a minimum to avoid excessive heating during magnetic field sweeps. A thermometer is mounted on the cell, in good thermal contact with the sample. (iv) Due to the bore size of the magnet, the maximum diameter of the cell is restricted to $32 \mathrm{~mm}$. This leaves a distance of $1.5 \mathrm{~mm}$ to the wall of the IVC on all sides. The sample space inside the cell is restricted to a diameter of 19 $\mathrm{mm}$ and a height of $7 \mathrm{~mm}$, a volume in which the magnetic field is homogeneous within $1 \%$. (v) The gas feedthrough of the cell is closeable by a miniature valve and the cell can be disconnected from the gas supply. In this way no highpressure gas tubing into the cryostat is necessary, which would increase significantly the base temperature. Moreover, the cell is easily transferred to other cryostats and experimental stations. (vi) The cell has a window, which allows illumination of the whole sample area. An optical fiber, 


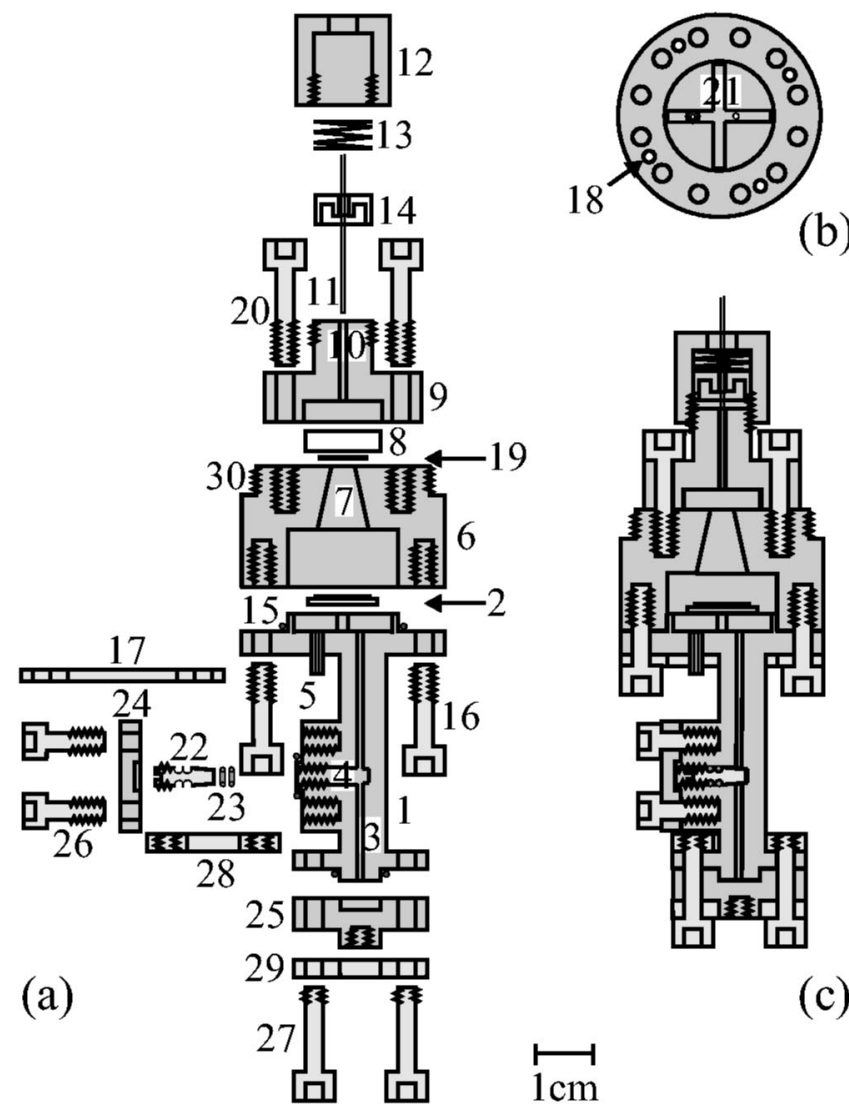

FIG. 1. Schematic of the high-pressure hydrogen loading cell. The grayscale indicates the materials used $(\mathrm{BeCu}-$ light gray; $\mathrm{Cu}$-gray; rubber-dark gray; In-black), zigzag lines symbolize threads, and numbers refer to the parts. (a) Cross sections of every part separately, (b) top view of the sample stage (1), and (c) cross section of the mounted cell.

which is a poor thermal conductor and thoroughly anchored, guides light from outside the ${ }^{3} \mathrm{He}$ flow cryostat to the cell window. This is advantageous over the use of, e.g., a cryoLED, since all illuminating optics are at RT and can be adjusted readily. (vii) The cell has a feedthrough for electrical wiring to perform conductivity and Hall measurements.

\section{CELL DESIGN}

Unless stated otherwise, all parts of the cell are made of high purity copper, which has been chosen for its combination of good thermal conduction at low $T$ and strength, at the cost of eddy current heating during magnetic field sweeps. The body of the cell consists of three main parts [where the numbering refers to Fig. 1(a)]. First is the sample stage (1), on top of which is mounted the sample (2). It incorporates a gas inlet (3), a miniature valve (4), and access for electrical wiring (5). Second is the lid (6) that covers the sample stage. It has a conical opening (7) to admit light without shadowing effects, while reducing the contact area of the window (8) with the hydrogen at high pressure to $4 \pi \mathrm{mm}^{2}$. Third is the clamp (9) required to press a window (Oriel Instruments fused silica window, thickness $3.5 \mathrm{~mm}, \oslash 12.7 \mathrm{~mm}$ ) against the lid. The cylindrical hole that holds the window has an exact depth of $3.5 \mathrm{~mm}$ but a diameter of $13.7 \mathrm{~mm}$, allowing for differential contraction of the clamp and the window, which otherwise would shatter upon cooling. Furthermore, the clamp has a feedthrough $(10, \oslash 640 \mu \mathrm{m})$ to guide a single, multimode optical fiber (11, Ocean Optics UV/VIS fused silica fiber with polyimide buffer, core diameter 600 $\mu \mathrm{m}$, stripped from its protective sleeves) to the window. The clamp has a thread at the top to screw a cap (12) that is loaded with a phosphor bronze spring (13). The spring is also constrained by a cylinder (14) that is rigidly attached to the fiber with epoxy (Stycast 2850 FT, catalyst 9). Thus rotating the cap puts the spring under tension such that it pushes the bare, polished end of the fiber against the window, regardless of thermal contractions during temperature sweeps. The light leaves the silica fiber in a cone with a half-angle of $12.7^{\circ}$ (equivalent to the numerical aperture $\mathrm{NA}=0.22$ of the fiber) and is hardly deflected by the silica window, which has the same index of refraction as the fiber. The conical opening in the lid with a half-angle of $12.8^{\circ}$ then allows illumination of a $13 \mathrm{~mm}$ diameter surface on top of the sample stage, which is at a distance of $18 \mathrm{~mm}$ from the window.

In order to assure leak-tight seals in the pressure range from $0.1 \mu$ bar to 200 bars at RT, we placed an indium O-ring (15) in the corner between the sample stage and the lid and tightly fixed these parts together with $12 \mathrm{BeCu}$ screws (16), fitted through two $\mathrm{BeCu}$ half-rings (17) that distribute the applied forces. Jacking screws are engaged in four-threaded holes [(18), see Fig. 1(b)] to separate these parts when changing samples. The O-ring is made by soldering together the ends of an appropriate length of In wire $(\oslash 1 \mathrm{~mm})$. By contrast, a solid O-ring (19, inner diameter $5 \mathrm{~mm}$, outer diameter $8 \mathrm{~mm}$ ) is cut from an In piece that is prepressed to a thickness of $0.1 \mathrm{~mm}$. This O-ring is applied as a seal between the window and the lid, after which the lid and clamp are permanently fixed together with six BeCu screws (20).

The feedthrough (5) for insulated copper wires is made of a nonmagnetic stainless steel pipe (type 316, inner diameter 0.025 in., wall thickness 0.035 in.), which is soft soldered to the cell and filled with Stycast 2850 FT. The epoxy mushrooms over the outer end of the pipe, providing a leaktight seal. Both the electrical feedthrough and the gas inlet end in a cruciform groove (21) on top of the sample stage [see Fig. 1(b)]. This groove allows the wires and the gas to enter under the sample and emerge at its side. It also houses four gold, miniature sockets (Microdot Twist/Con), which are attached in the groove with Stycast after the copper wires have been soldered (Oxford Instruments nonsuperconducting solder). The mating gold pins are soldered to similar copper wires that run to the sample, in our case a $500 \mathrm{~nm}$ thick Y film deposited in a UHV electron-gun deposition system on a $12 \times 12 \mathrm{~mm}^{2}$ sapphire $\mathrm{Al}_{2} \mathrm{O}_{3}$ substrate. These copper wires are glued to the substrate as strain relief, and at the other end attached with silver epoxy (Acheson Electrodag 1415) to four $7 \times 2 \mathrm{~mm}^{2}$ gold contact pads, predeposited on the substrate by sputtering. A gold wire $(\oslash 25 \mu \mathrm{m})$ is connected to each contact pad by ultrasonic wedge bonding. The other ends of these four gold wires are equally spaced on the perimeter of the circular Y film. The substrate is attached to the sample stage with a thin layer of GE 7031 varnish (Oxford Instruments), a reasonably good conductor at low $T$ and removable with a methanol/toluene mixture at RT. Thus the Y film is thermally anchored to the cell by way of the substrate 
and its electrical connections, the only thermal coupling left once the hydrogen gas in the sample space becomes liquid at $T \sim 22 \mathrm{~K}$ and freezes at $T \sim 14 \mathrm{~K}$.

The miniature valve consists of a $\mathrm{BeCu}$ plug (22) with threading and two circular grooves, each of which holds a Viton O-ring (23, Apple Rubber Products, 75 VT, inner diameter 0.079 in., outer diameter 0.125 in.) and puts it under appropriate tension. When the plug is in the open position during gas loading at RT, the Viton O-rings prevent leakage both under vacuum conditions and at pressures up to 200 bars, their performance remaining unaffected by thermal cycling. After loading, the plug is tightly fastened and its conical end (with a half angle of $5^{\circ}$ ) is engaged with the softer $\mathrm{Cu}$ counterpart in the sample stage, which accurately closes the gas inlet and prevents leakage down to the lowest $T$. Caps 24 and 25 are mounted for safety. After the cell is disconnected from the gas-loading system, these caps are fixed to the sample stage, each with six $\mathrm{BeCu}$ screws $(26,27)$ and an In O-ring as in case of the lid. Moreover, Cap 25 also houses a threaded hole to mount a copper block that accommodates two calibrated thermometers (Lakeshore Cryotronics carbon-glass resistor for $T \geqslant 2 \mathrm{~K}$ and Leiden Cryogenics carbon resistor for $T \leqslant 4 \mathrm{~K}$ ). This cap is clamped to the sample stage via four $\mathrm{BeCu}$ half rings $(28,29)$. Based on suggested allowable pressure tables for copper tubing ${ }^{3}$ we chose a minimum cell wall thickness of $3 \mathrm{~mm}$. The number of $\mathrm{BeCu}$ screws, their $3 \mathrm{~mm}$ outer diameter, $3 \mathrm{~mm}$ minimum engagement length, and $0.5 \mathrm{~mm}$ pitch are based on calculations of the maximum allowed load. ${ }^{4}$

\section{CELL PERFORMANCE}

In order to test the cell's strength we connected a water supply and applied 300 bar internal fluid pressure at RT. The fully mounted cell withstood the applied forces and was waterproof at this pressure. Next we connected a helium gas container and applied 200 bar internal gas pressure at RT. Leakage to the outside was not detected locally at any part of the cell up to the maximum sensitivity of a helium leak detector of $10^{-6} \mathrm{mbar} 1 / \mathrm{s}$. The final proof of the leak tightness of the cell for hydrogen gas pressures up to 200 bars is provided by measurements of the electrical conductivity $\sigma(\mathrm{RT})$ of the $\mathrm{YH}_{x}$ sample itself. After $\sigma(\mathrm{RT})$ has reached its equilibrium value during loading at a chosen pressure, it remains constant within the measurement accuracy when the valve is closed. Moreover, after cooling to base and warming to RT, $\sigma(\mathrm{RT})$ returns to its original value, indicating that no hydrogen entered or left the cell.

The electrical contact configuration described above allows us to perform accurate measurements of the absolute value of $\sigma$ and of the Hall voltage $V_{H}$ by the van der Pauw method. ${ }^{5}$ We measured the four-probe resistance of four, instead of two, van der Pauw configurations, and determine $\sigma$ in two independent ways. Thus we continuously monitor the homogeneity of the sample in the $a b$-direction and can follow the process of hydrogen loading: when both $\sigma$ values have converged and $d \ln \sigma / d \ln t<0.01$, hydrogen loading is stopped (where $t$ is time). The latter criterion is chosen for homogeneity along the $c$ axis and it is typically met 1 day

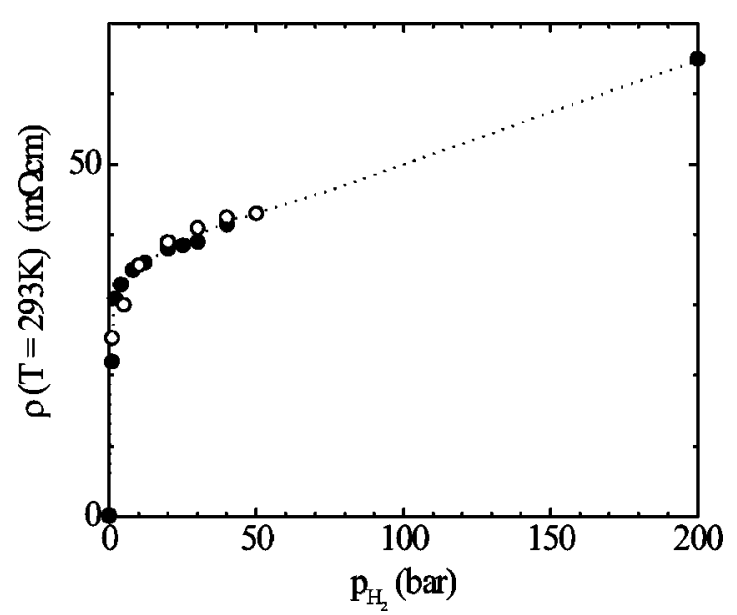

FIG. 2. Resistivity $\rho$ of a $500 \mathrm{~nm}$ thick $\mathrm{YH}_{x}$ film (covered by $5 \mathrm{~nm} \mathrm{Pd}$ ) as a function of applied hydrogen pressure $p_{\mathrm{H}_{2}}$ at room temperature (open symbols for loading, closed symbols for unloading, the dotted line is a guide to the eye).

after pressure has been applied. Using a turbomolecular pump (pumping speed $\sim 150 \mathrm{l} / \mathrm{s}$ at $10^{-6} \mathrm{mbar}$ ) we can only evacuate the sample space to a residual gas pressure of $\sim 10^{-3}$ mbar due to the diameter and length of the gas inlet ( 1 and $43 \mathrm{~mm}$, respectively). However, $p_{\mathrm{H}_{2}}$ in the sample space for loading pressures below 1 bar can be accurately determined and set on top of this offset, even in the $0.1 \mu \mathrm{bar}$ range, by monitoring the room temperature resistivity $\rho(\mathrm{RT}) \equiv 1 / \sigma(\mathrm{RT})$ of the sample and comparing its value with those from a $\rho$ versus $p_{\mathrm{H}_{2}}$ room temperature loading curve of an equivalent sample measured in a large container. ${ }^{6}$ Outside the cell, $p_{\mathrm{H}_{2}}$ is determined in the range from $0.1 \mu$ bar to 1 bar via absolutely calibrated and gas independent pressure gauges (MKS Baratron capacitance manometers 120AA-000 and 626A13MDE, full scale 0.1 bar and 1 bar, resolution 0.1 $\mu$ bar and 0.1 mbar, respectively) and in the range from 1 to 200 bars via dial gauges (Scott Specialty Gases, models 54$68 \mathrm{ES}-30 / 100 / 600 / 3 \mathrm{~K}$, full scale $2 / 7 / 40 / 200$ bar, accuracy $\pm 1 \%$ of full scale). In the $\mu$ bar range, $p_{\mathrm{H}_{2}}$ can be tuned with an accuracy better than $0.1 \mu$ bar using a double needle valve (Lakeview Valve \& Fitting, double metering valve, SS-SS2D-TVVHLL) and a dump vessel. Alternatively, one can apply partial hydrogen pressure by diluting dry nitrogen $\mathrm{N}_{2}$ with $\mathrm{H}_{2}$, which has the disadvantage of introducing extra residual gas in the sample space. We present in Fig. 2 the resulting resistivity $\rho$ versus $p_{\mathrm{H}_{2}}$ curve as measured with this setup at RT for the polycrystalline $\mathrm{YH}_{x}$ film during loading (closed circles) and unloading (open circles).

The base temperature of $312 \mathrm{mK}$ as measured by the $\mathrm{RuO}_{2}$ resistor at the ${ }^{3} \mathrm{He}$ pot is found to rise only $3 \mathrm{mK}$ when the cell and optical fiber are mounted. The bare fiber is fed into the IVC at the top of the ${ }^{3} \mathrm{He}$ insert through a hole $(\oslash 1$ $\mathrm{mm}$ ) in a vacuum flange, which is sealed with Stycast epoxy. The fiber is terminated at the RT side directly behind the flange with an SMA connector that can be capped to minimize heat radiation. The fiber is wrapped around the $1 \mathrm{~K}$ pot and the ${ }^{3} \mathrm{He}$ pot with copper foil and GE varnish for thermal anchoring. The lid of the cell is equipped with a thread [(30) 


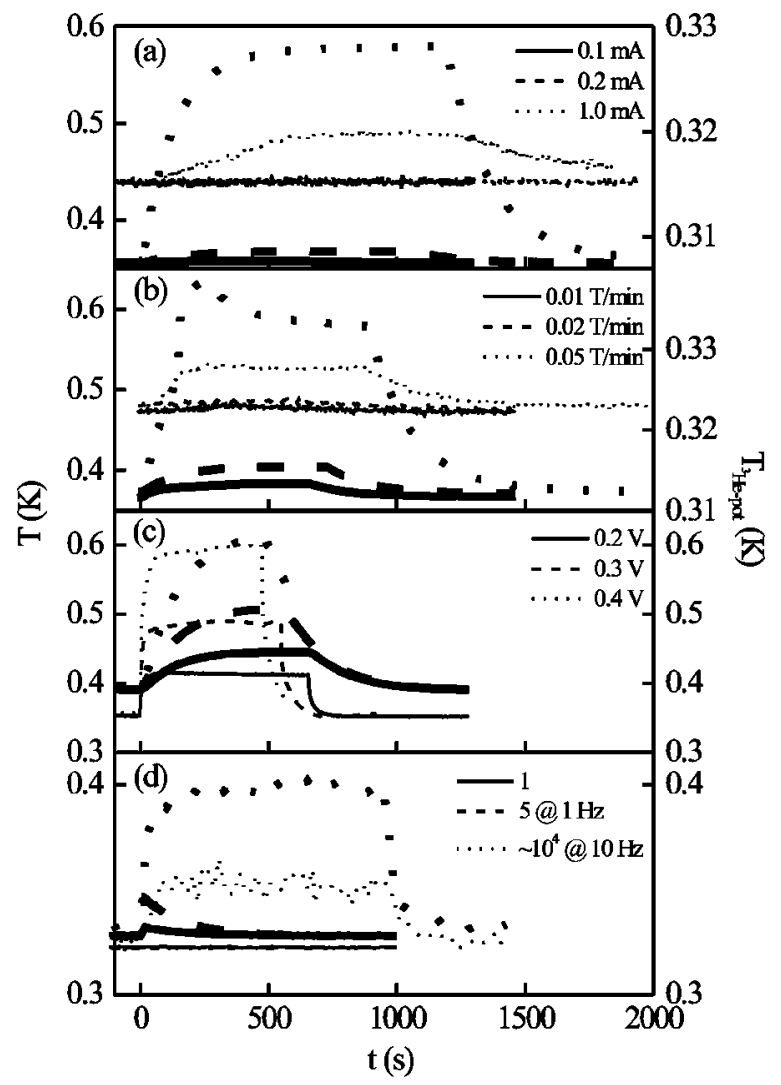

FIG. 3. Temperature $T$ of the cell (thick lines, left ordinate) and temperature of the ${ }^{3} \mathrm{He}$ pot $T_{3} \mathrm{He}$ pot (thin lines, right ordinate) simultaneously measured as a function of time $t$ under various thermal conditions. In (a) the current through the sample is changed, in (b) the magnetic field sweep rate is varied, in (c) the voltage across the heater on the ${ }^{3} \mathrm{He}$ pot is tuned, while in (d) the total number of pulses produced by a stroboscopic light source is adjusted.

in Fig. 1(a)] with which the cell can be screwed to the end of a cold tail. This extension consists of three silver rods $(\oslash 3$ $\mathrm{mm}$, length $12 \mathrm{~cm}$ ) that are soft soldered to the thread's copper counterpart on one side and to a copper disk on the other. This disk can then be tightly fastened to the ${ }^{3} \mathrm{He}$ pot.

The base temperature at the cell, as measured by the carbon resistor, is $351 \mathrm{mK}$, which represents an unwelcome temperature gradient $\Delta T=36 \mathrm{mK}$ to the ${ }^{3} \mathrm{He}$ pot. In order to establish its origin, we use the virgin sample as a heater [see Fig. 3(a)] and determine the overall thermal conductance $K_{T}$ of the cell and the cold tail. At these low temperatures, $K_{T}(T)=k_{0} T$, in which case the equilibrium heat transport equation reduces to

$$
\Delta T_{s} \cong \frac{p_{s}}{k_{0} T}
$$

for small thermal gradients. ${ }^{7}$ Here, $p_{s}=I^{2} \cdot R$, the power generated in the sample with resistance $R$, and $\Delta T_{s}$ is the corresponding temperature gradient from the cell to the ${ }^{3} \mathrm{He}$ pot (on top of $\Delta T$ ). We find $T \Delta T_{s}=1.3 \times 10^{5} \cdot I^{2}$ (open squares in Fig. 4), and with the residual resistance of the virgin sample $R(T \leqslant 15 \mathrm{~K}) \approx 0.35 \Omega$ we have $k_{0}=2.7 \times 10^{-6}$ $\mathrm{W} / \mathrm{K}^{2}$. In metals at low temperature, a linear dependence $K_{T} \propto T$ is associated with electronic thermal conductivity, in which case $k_{0}=\kappa_{0} A / L$, where $\kappa_{0}$ is the thermal conductivity constant of the metal, $A$ is the cross-sectional area through

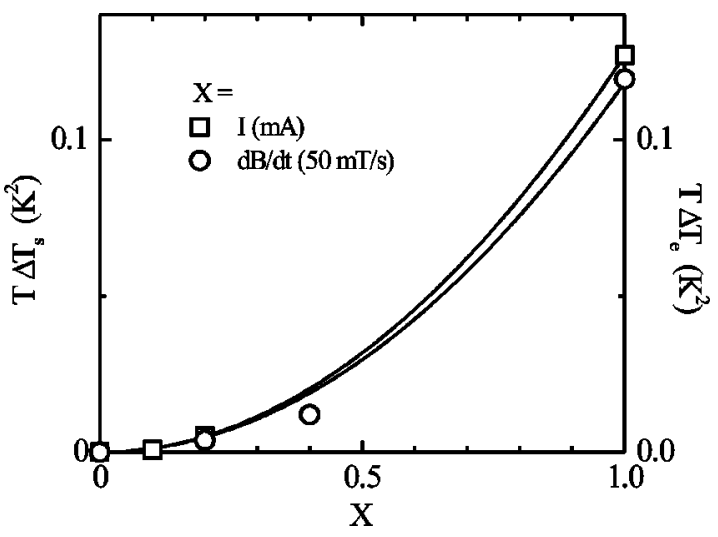

FIG. 4. The products $T \Delta T_{s}$ and $T \Delta T_{e}$ as a function of parameter $X$, at low $T$. Here $T$ is the temperature of the cell, $\Delta T_{s}$ is the temperature gradient between the cell and the ${ }^{3} \mathrm{He}$ pot due to heating by the sample (in which case $X$ is the current through the sample in $\mathrm{mA}$; open squares), and $\Delta T_{e}$ is the temperature gradient due to eddy current heating (in which case $X$ is the magnetic field sweep rate in $50 \mathrm{mT} / \mathrm{s}$; open circles). The quadratic dependence of these products on $X$ implies that the thermal conductance of the total system $K(T) \propto T$ at low $T$.

which the heat is transported, and $L$ is the distance from the heat source to the cold bath. If we take $L=12 \mathrm{~cm}$ and $A=21 \mathrm{~mm}^{2}$ (equivalent to the length and total crosssectional area of the three silver rods of the cold tail), then $\kappa_{0} \cong 0.2 \mathrm{~mW} / \mathrm{cm} \mathrm{K}^{2}$. This value is two orders of magnitude lower than that for pure $\mathrm{Ag},{ }^{7}$ which implies that the silver rods are not the bottleneck. Hence thermal boundary resistances must account for the low $k_{0}$ of this system.

The heat leak $p_{h}$ can now be estimated: $p_{h} \cong k_{0} T \Delta T$ $=40 \mathrm{nW}$. It can be due to radiation from the walls of the IVC or transferred by the fiber (although the fiber is an effective attenuator for wavelengths above $750 \mathrm{~nm}$ ), or arise from coupling to the helium bath via the residual exchange gas in the IVC. The slow conversion from ortho- to para- $\mathrm{H}_{2}$ of hydrogen absorbed in the $\mathrm{Cu}$ and $\mathrm{Ag}$ during production may contribute as well. In the case of a $10 \mathrm{ppm} \mathrm{H}_{2}$ contamination, a typical dissipation of $\sim 20 \mathrm{nW}$ in the $\sim 350 \mathrm{~g} \mathrm{Cu}$ of our cell could occur over a period of days. ${ }^{7}$ It can be prevented by decreasing the amount of $\mathrm{H}_{2}$ inclusions by annealing parts of the cell and cold tail in vacuum at elevated $T$. However, it is important to note that heat input due to orthoto-para conversion of frozen $\mathrm{H}_{2}$ in the sample space does not play a role, since it involves at most $\sim 0.1 \mathrm{~mol} \mathrm{H}_{2}$ (when loading at $p_{\mathrm{H}_{2}}=200 \mathrm{bar}$ and RT). This leads to dissipation only of order $10^{-2} \mathrm{nW}$. $^{7}$

We show in Fig. 3(b) the influence of eddy current heating at base $T$. Since $\sigma$ and $V_{H}$ in $\mathrm{YH}_{x}$ are nonhysteretic with $T$ and $H$, such heating does not form an insurmountable problem. Moreover, eddy current heating becomes increasingly less important at higher $T$. The thermal gradient $\Delta T_{e}$ from the cell to the ${ }^{3} \mathrm{He}$ pot due to eddy currents follows $T \Delta T_{e}=1.7 \times 10^{5} \cdot(d B / d t)^{2}$ (as can be seen in Fig. 4, open circles). This indicates that Eq. (1) can be used again, but now with $p_{s}$ replaced by $p_{e}=g V(d B / d t)^{2} / \rho$, the power generated by eddy currents in a conducting body with volume $V$, geometry factor $g$, and resistivity $\rho{ }^{7}$ Taking a residual resistivity $\rho=2 \mathrm{n} \Omega \mathrm{cm}$, as is typical for Cu with 20 ppm impurities, we find $g V \cong 1.7 \times 10^{5} \cdot k_{0} \cdot \rho=10^{-11} \mathrm{~m}^{5}$. This is two or- 
ders of magnitude smaller than expected for the effective cylindrical volume of the $350 \mathrm{~g} \mathrm{Cu}$ cell with radius $r$ $=1.5 \mathrm{~cm}$, height $5 \mathrm{~cm}$, and $g=r^{2} / 8$, implying that eddy currents are effectively damped by the real geometry of the cell and the higher resistivity of the other cell materials.

We plot in Fig. 3(c) the warming of the ${ }^{3} \mathrm{He}$ pot and the cell when increasing the voltage across the $70 \Omega$ heater on the ${ }^{3} \mathrm{He}$ pot. The ${ }^{3} \mathrm{He}$ pot reaches a new equilibrium within a minute, while the cell typically needs $\sim 10 \mathrm{~min}$ to stabilize. At higher $T$, the temperature gradient $\Delta T$ from the cell to the ${ }^{3} \mathrm{He}$ pot diminishes and disappears by $0.8 \mathrm{~K}$ due to the increased thermal conductivity of the cell and the cold tail. Finally, we show in Fig. 3(d) the effect of heating due to optical illumination at base $T$. Light pulses are produced by an ultraviolet stroboscope (Ocean Optics PX-1 pulsed xenon strobe lamp, spectral range $\sim 225-700 \mathrm{~nm}, 10 \mathrm{~Hz}$ pulse rate maximum, $0.54 \mathrm{~J}$ per pulse, $0.5 \mu$ s pulse width at $1 / 3$ pulse height). The output of the bulb is spatially not integrated nor focused, and only a fraction $\left(\sim 10^{-5}\right)$ of the produced light enters the optical fiber. During constant stroboscopic illumination at the maximum pulse rate the ${ }^{3} \mathrm{He}$ pot hardly warms up, but the temperature at the cell rises to $0.4 \mathrm{~K}$. Without a microscopic model for the persistent photoconductivity in $\mathrm{YH}_{x}$, apparently resulting from rearrangements of local bonding configurations, ${ }^{2}$ it is not possible to assess quantitatively the energy balance.

The high-pressure hydrogen loading cell described here can be used for a large variety of magnetotransport measure- ments under optical illumination. The cell's miniature valve, optical window, seals, and electrical feedthroughs are all leak tight at least down to a level of $10^{-6} \mathrm{mbar} \cdot 1 / \mathrm{s}$ for gas pressures up to 200 bars at room temperature, and its performance is unaffected by thermal cycling. At the lowest $T$ of the ${ }^{3} \mathrm{He}$ flow cryostat we find a thermal conductance $K_{T}(T)=2.7 \times 10^{-6} T$ for the cell and its suspension system. We expect that even lower $T$ can be reached with improved $K_{T}$, for example, when the cell is directly mounted to the plastic mixing chamber of a dilution refrigerator.

\section{ACKNOWLEDGMENTS}

The authors are indebted to H. Krebs for his sage advice and technical assistance. The work at The University of Chicago was supported by NSF Grant No. DMR-0114798.

${ }^{1}$ J. N. Huiberts, R. Griessen, J. Rector, R. Wijngaarden, J. Dekker, D. G. de Groot, and N. Koeman, Nature (London) 380, 231 (1996).

${ }^{2}$ A. F. Th. Hoekstra, A. S. Roy, T. F. Rosenbaum, R. Griessen, R. J. Wijngaarden, and N. J. Koeman, Phys. Rev. Lett. 86, 5349 (2001); D. Voss, Science 292, 5524 (2001).

${ }^{3}$ Swagelok Tubing Data, the Swagelok Companies, April 1989.

${ }^{4}$ E. Oberg and F. D. Jones, Machinery's Handbook, 18th ed. (Industrial, New York, 1968).

${ }^{5}$ L. J. van der Pauw, Philips Res. Rep. 13, 1 (1958); P. M. Hemenger, Rev. Sci. Instrum. 44, 698 (1973).

${ }^{6}$ J. N. Huiberts, Ph.D. thesis, Vrije Universiteit, 1995 (unpublished).

${ }^{7}$ F. Pobell, Matter and Methods at Low Temperatures (Springer-Verlag, Berlin, 1992). 